\title{
A Possible World Approach to Uncertain Relational Data
}

\author{
Ander de Keijzer \\ Faculty of EEMCS, University of Twente \\ POBox 217, 7500AE Enschede, The Netherlands \\ a.dekeijzer@utwente.nl
}

\author{
Maurice van Keulen \\ Faculty of EEMCS, University of Twente \\ POBox 217, 7500AE Enschede, The Netherlands \\ m.vankeulen@utwente.nl
}

\begin{abstract}
Data exchange between embedded systems and other small or large computing devices increases. Since data in different data sources may refer to the same real world objects, data cannot simply be merged. Furthermore, in many situations, conflicts in data about the same real world objects need to be resolved without interference from a user. In this paper, we report on an attempt to make a RDBMS probabilistic, i.e., data in a relation represents all possible views on the real world, in order to achieve unattended data integration. We define a probabilistic relational data model and review standard SQL query primitives in the light of probabilistic data. It appears that thinking in terms of 'possible worlds' is powerful in determining the proper semantics of these query primitives.
\end{abstract}

\section{Introduction}

Nowadays, notebooks, PDA's, cell phones, home networks, etc. have become commonplace. In recent years, all these systems have been equipped with mobile transmitters, enabling them to communicate with devices in their surroundings. As a result, ever more data is being exchanged to enable each device to obtain sufficient information about its environment (i.e., the real world). In this process, data originating from different data sources needs to be merged. An old and well-known problem is that data sources structure their data according to different schema's. Additionally, we may not even assume that these data sources agree on the data itself, i.e., that they carry conflicting information about the same real world objects. Somehow, these conflicts have to be resolved preferably without interference from a user.

The approach we take is to fundamentally drop the assumption that a database should contain accurate and complete data, but still hold the DBMS responsible for data management. Furthermore, it should be possible that data integration is performed in an unttended way, i.e., without interference from a user resolving conflicts. In other words, the DBMS should be able to manage uncertain data.
We propose a probabilistic RDBMS, built on top of a traditional relational database. We define a probabilistic relational data model and review standard SQL query primitives in the light of probabilistic data. It turns out that especially the semantics of aggregate operations is not trivial. Thinking of the database as holding information about possible worlds proved fundamental in defining a proper semantics for probabilistic SQL. Moreover, the probabilistic DBMS is designed as a proper extension of a traditional RDBMS.

Details for this research can be found in [1].

\section{Related Research}

The idea of a database dealing with uncertain or imperfect data is not new. For example, in the fields of artificial intelligence, deductive databases, knowledge discovery in databases, papers were regularly published on handling probabilistic data. Our approach to probabilistic data is that of worlds and interpretations. These possible worlds were introduced in [2] and allow for natural reasoning about uncertain data.

The notation for probabilistic relations we use, is inspired by [3], but in contrast with this system, we assume a closed world, i.e., complete knowledge of all possibilities with associated probabilities.

Redefinition of algebraic query operators has been proposed in [3, 4, 5, 6, 7], of which [4] uses a possible world approach. None of these, however, redefine aggregate functions to support probabilistic data.

Since, we will use our system for unattended data integration, the system has to be easy to use and be as simple as possible. Therefore, point-probabilities instead of complex values [7] are used to indicate the level of certainty,

For data integration, we assume the existence of a real world object to be certain whereby its appearance can be uncertain. This results in certain objects with uncertain attributes, or in database terms, certain rows and uncertain columns. This distinction between probabilities associated with rows (Type-1) and those associated with columns (Type-2) is introduced by [2]. 
Table 1. Probabilistic relation addressbook

\begin{tabular}{|c|c|c|}
\hline name & & room \\
\hline 'Ed' & [1.0] & 3122 \\
\hline 'Ed' & [1.0] & 3120 \\
\hline 'Harry' & [1.0] & 2023 \\
\hline 'Harry' & [1.0] & $2012 \quad[0.6]$ \\
\hline
\end{tabular}

\section{Probabilistic Data}

A real world object can be represented by a tuple $T \in$ $D_{1} \times \cdots \times D_{n}$, where $D_{i}$ is the domain of attribute $i$. A set of real world objects can then be described by a relation $R \in \mathbb{P}\left(D_{1} \times \cdots \times D_{n}\right)$. Finally, we represent a real world by a database $D B \in \mathbb{P P}\left(D_{1} \times \cdots \times D_{n}\right)$. Without loss of generality, we assume $D B$ to consist of one relation.

A probabilistic attribute is a traditional attribute with an associated probability, i.e., its domain is $\tilde{D}_{i}=[0,1] \times D_{i}$. We can now define a probabilistic tuple as $p T \in \tilde{D}_{1} \times \cdots \times$ $\tilde{D}_{n} . p T$ defines a possible description of a real world object, also called a possibility. Let $P_{i}(p T)$ be the probability part of attribute $i$ in tuple $p T$ and $\pi_{i}(p T)$ its value part.

Let $p R \in \mathbb{P}\left(\tilde{D}_{1} \times \cdots \times \tilde{D}_{n}\right)$ be a probabilistic relation. Table 1 shows an example of a probabilistic relation. We require $p R$ to have a primary key $k$ (or key for short). Without loss of generality, we assume the key of a probabilistic relation to consist of one attribute. Key $k$ uniquely identifies the real world object. As a consequence, the associated probability needs to be 1 . Observe that the key value itself is not unique in $p R$ in the traditional sense ('name' in Table 1).

For convenience, we introduce the following notation:

$$
\begin{aligned}
\bar{\Pi}_{v}(p R) & =\left\{p T \in p R \mid \pi_{k}(p T)=v\right\} \\
\left.D_{i}\right\rceil p R & =\left\{v \in D_{i} \mid(\exists p T \in p R) \bullet \pi_{i}(p T)=v\right\}
\end{aligned}
$$

$\bar{\Pi}_{v}(p R)$ is the set of all tuples from $p R$ where the key equals $\left.v . D_{i}\right\rceil p R$ is domain $D_{i}$ restricted to values occurring in $p R$.

Two constraints should hold for a probabilistic relation:

$$
\text { 1. } \left.\left(\forall v \in D_{k}\right\rceil p R\right) \sum_{p T \in \bar{\Pi}_{v}(p R)}\left(\prod_{1 \leq i \leq n} P_{i}(p T)\right)=1
$$

2. $\left.\left(\forall v \in D_{k}\right\rceil p R\right)(\forall i \neq k)\left(\forall p T, p T^{\prime} \in \bar{\Pi}_{v}(p R)\right) \bullet$ $\pi_{i}(p T)=\pi_{i}\left(p T^{\prime}\right) \Rightarrow P_{i}(p T)=P_{i}\left(p T^{\prime}\right)$

The first constraint states that for each real world object, the total probability of all possibilities equals one. This implies that we assume a closed world. The second constraint states that all attributes are independent.

\subsection{Possible Worlds}

If we consider a normal relation $R$ to be a representation of the real world, then we can consider a probabilistic re-
Table 2. Two addressbooks

\begin{tabular}{|l|l|l|l|}
\hline name & room & name & room \\
\hline 'Ed' & 3122 & 'Ed' & 3120 \\
\hline 'Harry' & 2023 & 'Harry' & 2012 \\
\hline
\end{tabular}

lation $p R$ to be a representation of several possible worlds. Since the probabilities of the individual attributes are independent, we obtain the probability of a description $p T$ of a real world object (i.e., a possibility) by multiplying the probabilities of the associated attributes: $\prod_{1 \leq i \leq n} P_{i}(p T)$.

The set of tuples representing one possibility for each real world object is called a possible world $p W \subseteq p R$, defined by

$$
\begin{aligned}
& \text { 1. } \left.\left(\forall v \in D_{k}\right\rceil p R\right) \bullet\left|\bar{\Pi}_{v}(p W)\right|=1 \\
& \text { 2. }(\forall p T \in p R)\left(\exists p T^{\prime} \in p W\right) \bullet \pi_{k}(p T)=\pi_{k}\left(p T^{\prime}\right)
\end{aligned}
$$

The first constraint ensures that only one possibility is contained in $p W$ for each real world object. The second constraint ensures that each real world object from $p R$ is contained in $p W$ at least once.

The set of all possible worlds, also called universe, is denoted by

$$
P W R_{p R}=\{p W \subseteq p R \mid p W \text { is a possible world }\}
$$

\section{Application context}

Our application context is the one of (mobile) devices exchanging data on their own. This, however, leads to the problem of merging possibly conflicting data on the same real world objects. If we merge two data sets, we need not only decide on the resulting data structure and how to convert both source data sets to this structure, but also on the level of confidence of the resulting data itself. We focus on the latter. Table 1 is an example of a possible merge result of the two relations from Table 2 .

The assigned probabilities represent the level of confidence on the attribute values. They are assumed to be determined by a mapping function, which will not be discussed here. Possible explanations or facturs influencing the probabilities in the table are that one of the addressbooks belongs to one of the persons mentioned, making his own address more reliable, or a date associated with the addressbooks, where the most recent one is considered more accurate.

Querying this new addressbook may obviously result in an uncertain answer. That is the unavoidable consequence of unattended data integration. We believe that the moment a user views information, is the right moment for him/her to decide among possibilities. This decision can be fed back in the data by deleting possibilities and updating probabilities. 
Table 3. Addressbook with distances

(a) non-1NF representation

\begin{tabular}{|c|c|c|c|}
\hline name & room & & \\
\hline \multirow[t]{2}{*}{$\overline{\text { 'Ed' }}$} & 3122 & 20 & [0.7] \\
\hline & 3120 & 18 & {$[0.3]$} \\
\hline \multirow[t]{2}{*}{ 'Harry' } & 2023 & 50 & {$[0.4]$} \\
\hline & 2012 & 60 & [0.6] \\
\hline
\end{tabular}

(b) $1 \mathrm{NF}$ representation

\begin{tabular}{|l|l|l|l|l|}
\hline name & room & room_prob & distance & distance_prob \\
\hline 'Ed' & 3122 & 0.7 & 20 & 0.7 \\
\hline 'Ed' & 3122 & 0.7 & 18 & 0.3 \\
\hline 'Ed' & 3120 & 0.3 & 20 & 0.7 \\
\hline 'Ed' & 3120 & 0.3 & 18 & 0.3 \\
\hline 'Harry' & 2023 & 0.4 & 50 & 0.4 \\
\hline 'Harry' & 2023 & 0.4 & 60 & 0.6 \\
\hline 'Harry' & 2012 & 0.6 & 50 & 0.4 \\
\hline 'Harry' & 2012 & 0.6 & 60 & 0.6 \\
\hline
\end{tabular}

(c) $3 \mathrm{NF}$ representation

\begin{tabular}{|l|l|} 
MAIN & \\
\hline$\underline{\text { name }}$ & id \\
\hline 'Ed' & 1 \\
\hline 'Harry' & 2 \\
\hline
\end{tabular}

\begin{tabular}{|l|l|l|} 
ROOM & \multicolumn{2}{|l}{} \\
\hline$\underline{\mathrm{id}}$ & $\underline{\text { room }}$ & prob \\
\hline 1 & 3122 & 0.7 \\
\hline 1 & 3120 & 0.3 \\
\hline 2 & 2023 & 0.4 \\
\hline 2 & 2012 & 0.6 \\
\hline
\end{tabular}

\begin{tabular}{|l|l|l|} 
DISTANCE & \multicolumn{2}{|l}{} \\
\hline$\underline{\mathrm{id}}$ & $\underline{\text { distance }}$ & prob \\
\hline 1 & 20 & 0.7 \\
\hline 1 & 18 & 0.3 \\
\hline 2 & 50 & 0.4 \\
\hline 2 & 60 & 0.6 \\
\hline
\end{tabular}

\section{Storage scheme}

We developed a prototype system on top of an ordinary RDBMS. Probabilistic tables can be stored in several ways corresponding with the normal form to which one likes to adhere. Table 3 shows different representations of the same probabilistic relation addressbook: a representation with non-atomic attributes (Table 3(a)), the representation brought in first normal form (Table 3(b)), and the representation brought in third normal form (Table 3(c)). The 1NF representation of probabilistic data, however, may lead to massive replication of attribute values, hence, data inconsistency. The 3NF representation, on the other hand, may require many joins, hence, may perform less efficiently. We will discuss both representations.

\subsection{NF representation}

A probabilistic attribute $\tilde{D}$ is not atomic, since it consists of a probability part and a value part. The representation of a probabilistic relation $p R \in \mathbb{P}\left(\tilde{D}_{1} \times \cdots \times \tilde{D}_{n}\right)$ can be transformed to be represented only by atomic attributes. We write $p R \in \mathbb{P}\left([0,1] \times D_{1} \times \cdots \times[0,1] \times D_{n}\right) . p R$ is now in $1 \mathrm{NF}$.

Probability attributes which have only values of 0 or 1 are omitted, since these are not probabilistic, which is the case for at least the key of the relation.

If we bring Table 3(a) in 1NF, this results in $2 \times 2+2 \times$ $2=8$ rows (see Table $3(\mathrm{~b})$ ). Each row has 5 attributes: name, room, room_prob, distance and distance_prob.

\subsection{NF representation}

The $1 \mathrm{NF}$ representation may massively replicate attribute values, hence may lead to data inconsistency if not handled properly. Normalization to $3 \mathrm{NF}$ prevents this.

We start by observing that the probabilistic relation in Table 3(a) is modeled as $p R \in D_{k} \times \mathbb{P} \tilde{D}_{2} \times \cdots \times \mathbb{P} \tilde{D}_{n}$. Each non-atomic attribute can, however, be modeled as a separate table. Therefore, we introduce a main table for each probabilistic relation with the key and a unique identifier $D_{k^{\prime}}$ (to avoid replicating the key value). Furthermore, we introduce a separate table $D_{k^{\prime}} \times D_{i} \times[0,1]$ for each non-atomic attribute. The key of these tables consists of the first two attributes, $k^{\prime}$ and $i$.

The main table has functional dependency $k \mapsto k^{\prime}$ and the separate tables have functional dependencies $k^{\prime} i \mapsto$ $p$, where $p$ is the probability attribute with domain $[0,1]$. These functional dependencies satisfy $3 \mathrm{NF}$. The $3 \mathrm{NF}$ representation of table 3 is shown in table 3(c).

\subsection{Dependent attributes}

The attributes room and distance in table 3 are independent, which means that any given distance can occur with any given room. This becomes extra clear with the $3 \mathrm{NF}$ representation where both attributes are stored in different tables. In our example however, the distance is likely to depend on the room. The $3 \mathrm{NF}$ representation can model this dependency by storing dependent attributes in the same table. The attributes room and distance in the main table are then replaced by a single attribute called room_distance.

\section{Querying}

A probabilistic query should return a different kind of result than a normal relational query. Consider a query such as the one posed below on the addressbook of Table 1 . 
SELECT name, room

FROM addressbook

Seen from a traditional relational point of view, the answer \{('Ed', 3122), ('Ed', 1320), ('Harry', 2023), ('Harry', 2012)\} makes no sense: because attribute name is a key, only one tuple containing the name 'Ed' is expected. From the perspective of probabilistic relations, however, the returned result is a somewhat correct answer, because uncertainty about rooms in the data will inevitably lead to uncertainty in the answer to a question about rooms. The answer only does not specify the probabilities, which it should.

This calls for a review of the semantics of relational algebra operators and, as a consequence, their redefinition, in order to support querying on probabilistic data. With the possible world approach, we would expect the answer $\{(0.7$, 'Ed', 3122), (0.3, 'Ed', 3120), (0.4, 'Harry', 2023), (0.6, 'Harry', 2012)\}, which indicates that there is a $70 \%$ chance the possibility ('Ed', 3122) is correct and a 30\% chance the possibility ('Ed', 3120) is correct.

\subsection{Operators}

Select The traditional select operator $\sigma_{c}(R)$ selects tuples from $R$ according to the selection condition $c$. For a probabilistic select operator, the selection condition $c$ can also refer to probabilities of attributes. We, therefore, introduce a function $P(f)$ which returns the probability of attribute $f$. The semantics of the probabilistic select operator $\sigma_{c}(p R)$ should be that it selects possibilities from $p R$.

Note, however, that the result of a selection corresponds to a different universe than the one stored in the database. All probabilities of attributes $f$ in the answer are of the form $P(f \mid c)$. For example, to obtain data that is highly likely from the addressbook relation, highly likely being a probability more than $60 \%$, we could write $\sigma_{P(\text { room })>0.6}($ addressbook $)$. The result of this expression is $\{(1.0$, 'Ed', 3122) $\}$, which states that in the universe where a probability of a room is higher than $60 \%$, Ed certainly occupies room 3122 , hence the probability 1.0 in the answer. In other words, the original probabilities have to be normalized in the result. Note that this is a proper probabilistic relation according to Section 3. Furthermore, observe that dependency among attributes may occur.

Project The traditional project operator $\pi_{f}(R)$ selects all attributes $f$ from all tuples in $R$ leaving out other attributes not in $f$. The semantics of a probabilistic project operator $\pi_{f}(p R)$ should be that it selects all attributes $f$ from all possibilities in $R$. Since all attributes are independent, projection does not affect the associated probabilities. However, if the key of $p R$ is projected out, the individual objects can no longer be identified and the result is meaningless. Therefore, $k$ always has to be part of attribute list $f$.
Table 4. Determining the probabilities associated with a union result by enumerating possible worlds $(\breve{P}=1-P)$.

\begin{tabular}{|c|c|c|c|}
\hline \multicolumn{2}{|c|}{$A$} & $B$ & $A \cup B$ \\
\hline \multicolumn{2}{|c|}{$e \in A\left[P_{A}\right]$} & $e \in B\left[P_{B}\right]$ & $e \in A \cup B\left[P_{A} P_{B}\right]$ \\
\hline \multicolumn{2}{|c|}{$e \in A\left[P_{A}\right]$} & $e \notin B\left[\breve{P}_{B}\right]$ & $e \in A \cup B\left[P_{A} \breve{P}_{B}\right]$ \\
\hline \multicolumn{2}{|c|}{$e \notin A\left[\breve{P}_{A}\right]$} & $e \in B\left[P_{B}\right]$ & $e \in A \cup B\left[\breve{P}_{A} P_{B}\right]$ \\
\hline \multicolumn{2}{|c|}{$e \notin A\left[\breve{P}_{A}\right]$} & $e \notin B\left[\breve{P}_{B}\right]$ & $e \notin A \cup B\left[\breve{P}_{A} \breve{P}_{B}\right]$ \\
\hline \multirow[t]{2}{*}{$\Rightarrow$} & \multicolumn{3}{|c|}{$e \in A \cup B\left[P_{A}+P_{B}-P_{A} P_{B}\right]$} \\
\hline & \multicolumn{3}{|c|}{$e \notin A \cup B\left[1-\left(P_{A}+P_{B}-P_{A} P_{B}\right)\right]$} \\
\hline
\end{tabular}

Union The probabilistic union operator merges two probabilistic relations possibly containing possibilities for the same real world objects. To properly calculate the probabilities in the answer, it is beneficial to enumerate the possible worlds, i.e., consider each possibility of an element existing or not in the operand sets (see Table 4). The probability of the element occurring in the result is the sum of the three possibilities where the element occurred in either operand.

The intersection and difference can be determined analogously.

Cartesian product The semantics of a cartesian product is the simultaneous occurrence of two possibilities. Probabilities can be calculated according to this semantics.

\subsection{Aggregate functions}

Aggregate functions combine the values of a set of attribute values into one value. Examples are the total price of a collection or the average mark of students. Observe that a traditional aggregate operator works in one world where everything is certain. To come up with a proper semantics for aggregate operators in the context of probabilistic relations, we use the strategy of enumerating possible worlds. For each possible world $p W$ with associated probability $P(p W)$, we apply the traditional aggregate operator aggr. The results $(\operatorname{aggr}, P(p W))$ together form the resulting probabilistic relation.

Given the addressbook relation of Table 3 and the query below:

SELECT MAX (distance)

FROM addressbook

This would traditionally return the number 60 . This is, however, only true for the world where 'Harry' occupies room 2012, but there are three other possible worlds. These 
possible worlds should contribute to the result. The correct result

$$
\begin{aligned}
& \{(0.28,50),(0.42,60),(0.12,50),(0.18,60)\} \\
& \quad=\{(0.4,50),(0.6,60)\}
\end{aligned}
$$

reflects the existence of four possible worlds, two possibilities for both 'Ed' and 'Harry', each with its own maximum distance either 50 or 60 . The associated probabilities of worlds with equal maximum values can be combined as shown.

In more general terms, the probabilistic aggregate operators (MAX, MIN, SUM, AVG) are defined by

$$
\operatorname{aggr}_{f}(p R) \in \mathbb{P}([0,1] \times \mathbb{R})
$$

where $\mathrm{f}$ indicates a field name. We use the notation $\overline{a g g r}_{f}(p W)$ for the traditional counterpart of an aggregate operator evaluated in possible world $p W$. The aggregate function $a g g r_{f}$ is defined by

$$
\operatorname{aggr}_{f}(p R)=\left\{\left(P(p W), \overline{\operatorname{aggr}}_{f}(p W)\right) \mid p W \in P W R_{p R}\right\}
$$

A more elaborate explanation of the aggregate functions can be found in [1].

\subsection{EXP function}

All aggregate operators take the existence of possible worlds into account. However, the system should be able to predict information about the real world. We, therefore, introduce a new aggregate function, EXP, which returns the expected value of a numerical field.

$$
E X P_{f}(p R) \in \mathbb{R}
$$

It is defined by

$$
\frac{\sum_{p T \in p R} \pi_{f}(p T) \times P_{f}(p T)}{\sum_{p T \in p R} P_{f}(p T)}
$$

The function calculates the weighted average of the field over all possible worlds. In the presence of a GROUP BY clause, we assume $p R$ to represent one group. For example,

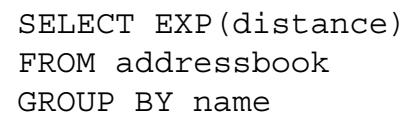

returns $\{19.4,56\}$, the expected distances to all persons in our addressbook.

EXP can be used in combination with other aggregate functions. The expected maximum distance is obtained by

SELECT EXP (MAX (distance))

FROM addressbook

\section{Conclusions}

We designed an extension to an ordinary RDBMS to allow it to handle uncertain data. Uncertain data is represented by probabilistic relations, relations that hold information on all possibilities of real world objects with associated probabilities. We assume a closed world. Storing probabilistic relations in an ordinary RDBMS proved simple by properly applying normal form theory on the functional dependencies related to probabilities.

To be able to properly query the data in a probabilistic database, we reviewed the semantics of all operators and redefined them to result in probabilistic answers. The approach of enumerating possible worlds proved crucial in choosing the right semantics for probabilistic operators. We also introduced a new aggregate operator EXP that returns the expected value. It can be used in prediction queries.

\section{References}

[1] Ander de Keijzer and Maurice van Keulen. A probabilistic database extension. Technical report, CTIT, TR-04-21, 2004.

[2] E Zimanyi and A Pirotte. Imperfect information in relational databases. Uncertainty Management in Information Systems, A. Motro and P. Smets, Eds., 1997.

[3] Daniel Barbará, Hector Garcia-Molina, and Daryl Porter. A probabilistic relational data model. In François Bancilhon, Costantino Thanos, and Dennis Tsichritzis, editors, Advances in Database Technology - EDBT'90. International Conference on Extending Database Technology, Venice, Italy, March 26-30, 1990, Proceedings, volume 416 of Lecture Notes in Computer Science, pages 60-74. Springer, 1990.

[4] Laks V. S. Lakshmanan, Nicola Leone, Robert Ross, and V. S. Subrahmanian. ProbView: a flexible probabilistic database system. ACM Transactions on Database Systems, 22(3):419-469, 1997.

[5] Debabrata Dey and Sumit Sarkar. A probabilistic relational model and algebra. ACM Trans. Database Syst., 21(3):339-369, 1996.

[6] Norbert Fuhr and Thomas Rölleke. A probabilistic relational algebra for the integration of information retrieval and database systems. ACM Transactions on Information Systems, 15(1):32-66, 1997.

[7] Thomas Eiter, Thomas Lukasiewicz, and Michael Walter. A data model and algebra for probabilistic complex values. Annals of Mathematics and Artificial Intelligence, 33(2-4):205-252, 2001. 